\title{
Prevalence of intestinal parasitic infection in food handlers of Iran: A Systematic Review and meta-analysis
}

\author{
Khojasteh Sharifi-Sarasiabi ${ }^{1}$, Mehrgan Heydari -Hengami ${ }^{1}$, Azar Shokri ${ }^{2}$, and Saeed \\ Hosseini Teshnizi ${ }^{3}$ \\ ${ }^{1}$ Hormozgan University of Medical Sciences \\ ${ }^{2}$ North Khorasan University of Medical Sciences \\ ${ }^{3}$ Affiliation not available
}

July 16, 2020

\begin{abstract}
Food handlers regardless whether preparing or serving food, key roles in transmission of food-borne infections. This study aimed to evaluate the prevalence of intestinal parasitic infections in food handlers. In present study, a comprehensive literature search was carried out in electronic databases including: PubMed, Scopus, Google Scholar, Science Direct, Magiran, Scientific Information Database (SID), Iran Medex and Iran Doc to identify all the published studies from 2000 to 31 st April, 2019. A total of 25 articles from different regions of Iran identified and fulfilled our eligibility criteria. Totally 140447 cases were examined and 1163 cases were infected with intestinal parasites. Of all cases, 19516 were male and 5901 were female with 1163 and 652 infected case respectively. The overall prevalence of intestinal parasitic infections was evaluated 14\% [95\%CI: 11$17 \%$ ]. Results indicated that protozoan such as Giardia lamblia, with prevalence of $41.0 \%$ [95\%CI: 25.0\%-59.0\%], Blastosystice hominis with $28 \%$ [95\%CI: $15.0 \%-44.0 \%$ ] and Entamoaba coli with $22.0 \%$ [95\%CI: $16.0 \%-29.0 \%$ ] had the highest prevalence while, Dientamoeba fragilis 5.0\% [95\%CI: 4.0\%-7.0\%], Iodamoeba. bütschlii 5.0\% [95\%CI: 2.0\%-8.0\%], Chilomastix mesnili 5.0\% [95\%CI: 2.0\%-9.0\%] and Endolimax. nana with 3.0\% [95\%CI: $1.0 \%-7.0 \%$, were less prevalent. Infection with Ascaris lumbricoides $7.0 \%$ [95\%CI: $0.0 \%-29.0 \%$ ] was more prevalent helminthes followed with Enterobius vermicularis 3.0\% [95\%CI: 1.0\%-5.0\%], Hymenolepis nana 2.0\% [95\%CI: 1.0\%-3.0\%], Taenia spp 2.0\% [95\%CI: 0.0\%-7.0\%] and Trichuris. trichiura $1.0 \%$ [95\% CI: $0.0 \%-1.0 \%]$. Our results revealed the high prevalence of intestinal parasitic infection in food handlers in Iran. Monitoring programs to prevent and controlling of transmission to individuals are needed
\end{abstract}

\section{Abstract}

Food handlers regardless whether preparing or serving food, key roles in transmission of food-borne infections. This study aimed to evaluate the prevalence of intestinal parasitic infections in food handlers. In present study, a comprehensive literature search was carried out in electronic databases including: PubMed, Scopus, Google Scholar, Science Direct, Magiran, Scientific Information Database (SID), Iran Medex and Iran Doc to identify all the published studies from 2000 to $31^{\text {st }}$ April, 2019. A total of 25 articles from different regions of Iran identified and fulfilled our eligibility criteria. Totally 140447 cases were examined and 1163 cases were infected with intestinal parasites. Of all cases, 19516 were male and 5901 were female with 1163 and 652 infected case respectively. The overall prevalence of intestinal parasitic infections was evaluated 14\% [95\%CI: 11-17\%]. Results indicated that protozoan such as Giardia lamblia, with prevalence of $41.0 \%$ [95\% CI: $25.0 \%$ 59.0\%], Blastosystice hominis with 28\% [95\%CI: 15.0\%-44.0\%] and Entamoaba coli with 22.0\% [95\%CI: 16.0\%-29.0\%] had the highest prevalence while, Dientamoeba fragilis 5.0\% [95\%CI: 4.0\%-7.0\%], Iodamoeba. bütschlii5.0\% [95\%CI: 2.0\%-8.0\%], Chilomastix mesnili 5.0\% [95\%CI: 2.0\%-9.0\%] and Endolimax. nana with 3.0\% [95\%CI: 1.0\%-7.0\%], were less prevalent. Infection withAscaris lumbricoides $7.0 \%$ [95\%CI: 0.0\%-29.0\%] was more prevalent helminthes followed with Enterobius vermicularis 3.0\% [95\%CI: 1.0\%-5.0\%], Hymenolepis 
nana 2.0\% [95\%CI: 1.0\%-3.0\%], Taenia spp 2.0\% [95\%CI: 0.0\%-7.0\%] and Trichuris. trichiura 1.0\% [95\%CI: $0.0 \%-1.0 \%]$.

Our results revealed the high prevalence of intestinal parasitic infection in food handlers in Iran. Monitoring programs to prevent and controlling of transmission to individuals are needed.

Keywords: Intestinal, Parasitic Infection, Food handler, Iran

\section{INTRODUCTION}

Intestinal parasitic infections are widespread in the world and transmitting directly or indirectly among populations. In some cases, carriers without any symptoms of disease are the main source of infection especially if they work as food handlers. given the high prevalence of 2 billion chronically infected individuals in the world, this factis not from the reality. The importance of this issueemerges when those people work as food handlers and do not care personal hygiene (Saki et al., 2012).

Although people are in constant contact with environmental pathogens including parasites, they are not affected seriously sincethe immunity is important in disease etiology. Despite the well toleration of parasitic infection in most of the healthy individuals, some people are vulnerable against parasites. The importance of parasitic infection ishighlighted when the infected individualplays a major role in food handling or food industries.

Iran is a suitable region for most of parasitesgrowth and distribution due to the geographic, socioeconomic and behavioral conditions. Serious efforts tocontrol parasitic infection have resulted in burden decrease of parasitic infections, but contamination with intestinal parasites is still a concern for health care services $(\mathrm{Ku}-$ solsuk et al.,2011). Using animal and human feces asfertilizersfor agricultures and vegetable gardens, climatic conditions, traditions and customs are consideredthe main reasons for the incidence of parasitic infections in some parts of the country. Direct transmission from person to person is another factor which complicates the parasite control programs. This kind of parasite transmission is markedly important in food handlers and particularly in oral-fecal parasites such as Giardia lamblia, Hymenolepis nanaand Enterobius vermicularis (Kusolsuk et al.,2011; Kheirandish et al.,2014). If food handlers do not care about personal hygiene, they can contaminate dishes, salad and other food materials which finally results in the contamination of the customers (Koohsaret al.,2012). Studies on transmitted parasites by food handlers indicate that Entamoeba coli is the most common nonpathogenic protozoa indicating contamination with fecal materials and poor hygiene (Kassani et al., 2015). There is a doubt about pathogenesis of some protozoan such as Blastocystis hominis, but the presence of it in large number may be associated with diarrhea (Motazedian et al., 2016).

Several studies have been conducted in different parts of the world regarding the prevalence of intestinal parasites in food handlers (Esparar et al., 2004). In this study, we performed a systematic review and metaanalysis to find out the pooled estimate of prevalence of intestinal parasites such as G. lamblia, E. coli , $B$. hominis and $H$. nana in food handlers, so the healthcare officials discovered the routs to prevent and control the disease transmitted by parasites and also the best and most practical method used in conducting experiments to achieve the best results.

\section{MATERIALS AND METHODS}

This systematic review and meta-analysis conducted based on the guidelines of Preferred Reporting Items for Systematic Reviews and Meta-Analyses (PRISMA) statement. (http://www.prisma-statement.org). The PROSPERO registration number is: CRD42019123662

\subsection{Literature search and Search strategy}

In this meta-analysis, a comprehensive literature search was carried out in electronic databases including: PubMed, Scopus, Google Scholar, Science Direct, Magiran, Scientific Information Database (SID), Iran Medex and Iran Doc identify all the published studies from 2000 to $31^{\text {st }}$ April, 2019. Duplicates, studies out of Iran were excluded. All original descriptive studies (designated as cross-sectional) about intestinal parasites in food handlers were concerned. The process is shown in Figure 1. The search was performed 
using terms: "intestinal parasites", "parasitic infection", "parasitic diseases", "parasite", "food handlers"," prevalence", alone or in combination, both in Persian and Englishlanguages.

\section{Data collection}

In the initial search of collected bibliographic references, 433 studies were founded. After removing duplicated, irrelevant studies and studies out of Iran, finally, 25 papers with epidemiological parameters of interest were fulfilled the inclusion criteria. Those articles reporting the prevalence of intestinal parasitic infections in food handlers in Iran were included to our study (Table 1).

\section{Data extraction}

Two authors screened the titles, abstracts, and full text of literatures, independently. Any disagreements between two reviewers were resolved by discussion among researchers. Extracted data were including: first author, the year of publication, prevalence rate, demographic information (age, gender), geographical region of study, diagnostic test, sample size (number of examined people) and the number of infected cases (Table $1)$.

1. Quality of studyTo assess the quality of observational studies included in this meta-analysis using a check list as in Table1. It contains 12 items with scores "Yes $=1$ " and "No=0". The sum of scores is 0 to 12 and for including study in meta-analysis a quality score of at least 8 is required.

\section{Statistical analysis}

After extracting the sample size and the number of positive infections for each study, the proportion of infection and standard error (se) were computed. Before estimating pooled effect size, sensitivity analysis used to explore the effect of each study on pooled effect size. Heterogeneity among studies assessed using both Q-test which suggestion by the Cochrane Handbook ( $<0.1$ as substantial heterogeneity) and I-square index $\left(I^{2}<50 \%\right.$, as substantial heterogeneity). If found that a substantial heterogeneity, sub group metaanalysis (fixed or random effect model) performed to compute the pooled prevalence of infection based on characteristic such as sex, country, education, pathogen and parasite species. In addition to meta-regression examined to find source of heterogeneity. To detect sources of heterogeneity we performed meta-regression on publish year and sample size of studies.

To evaluate publication bias, we aided a funnel plot and egger's test as a statistical test ( $\mathrm{p}<0.1$ as significant). If detected a substantially publication bias, the trim and fill method applied to estimate and adjust for the number of missing studies (due to publication bias) in a meta-analysis (Ebrahim ,2006). All statistical analysis performed using Stata/MP (version 14.0, College Station, TX, USA).

\section{RESULTS AND DISCUSSION:}

Among all eight databases searched and unpublished data from 2000 to 2019 (19 years), 25 articles were eligible to include in this systematic review and meta-analysis. The literature searches and selection process are shown in Figure 1. Totally 140447 cases were examined and 1163 cases were infected with intestinal parasites. Of all examined cases, although all studies did not define male and female genders, in studies which defined the gender of participants, a number of 19516 cases were male and 5901 cases were femalewith1163 and 652 infected cases respectively (Table 1). There was no significant difference between infection among males $13 \%(10 \%-15 \%)$ and females $8 \%(5 \%-11 \%)(P=0.39)$ and in age of participants $(\mathrm{P}=0.65)$ (Table 2).

To evaluate the effect of each study on the pooled estimate of prevalence, by repeating the meta-analysis after omitting each study, the sensitivity of studies was depicted in figure2. All effect sizes of 25 studies were located in 95\%confidence interval (95\%CI). Therefore, none of studies substantially had effect on pooled prevalence of intestinal infection and we can include all studies in meta-analysis (Figure2).

The results of Egger' test showed that there no evidence of publication bias among studies on spices of parasite $(\mathrm{p}>0.1)$. Also, there was not enough studies for assessing publication bias for Dientamoeba fragilis and Trichuris trichura (Table 3 ). 
The overall prevalence of intestinal parasitic infections in food handlers in Iran was evaluated 14\% [95\%CI: 11-17\%]. According to the results of subgroup analysis, Giardia lamblia, with prevalence of $41.0 \%$ [95\%CI: 25.0\%-59.0\%], Blastosystis hominis, with 28\% [95\%CI: 15.0\%-44.0\%] and Entamoaba coli with $22.0 \%$ [95\% CI: $16.0 \%-29.0 \%]$, had the highest prevalence, respectively. Also, other species had the prevalence between $1.0 \%$ (Trichuris trichiura) to $9.0 \%$ (Entamoaba histolytica /dispar ) (Figure 3).

The subgroup analysis for intestinal protozoan parasites revealed the prevalence of Dientamoeba fragilis 5.0\% [95\%CI: 4.0\%-7.0\%], Iodamoeba bütschlii 5.0\% [95\%CI: 2.0\%-8.0\%], Chilomastix mesnili 5.0\% [95\%CI: 2.0\%-9.0\%] and Endolimax nana 3.0\% [95\%CI:1.0\%-7.0\%]. The results for intestinal helminthic infections showed that Ascaris lumbricoides with prevalence of $7.0 \%$ [95\%CI: 0.0\%-29.0\%] had the highest prevalence and then Enterobiu svermicularis with infection rate of 3.0\% [95\%CI: 1.0\%-5.0\%],Hymenolepis nana with 2.0\% [95\%CI:1.0\%-3.0\%], Taenia spp with 2.0\% [95\%CI: 0.0\%-7.0\%] and Trichuris trichiura 1.0\% [95\%CI: 0.0\%-1.0\%] were the most prevalent intestinal helminthic infections (Figure 3). The highest rate of infection was found in owners of school cafeteria with $27.7 \%$ followed with $11.50 \%$ in butchers and $10.17 \%$ among bakeries. The lowest infection rate was $1.70 \%$ in confectioners (Table 4,5). The results of our analysis indicated that frequency of intestinal parasitic infections was in contrast with educational levels and increase of educational level, the intestinal parasitic infection was showed a decrease process. The prevalence of intestinal parasitic infection in individuals with education level lower than high school, was estimated $20 \%$ [95\%CI:9.0-34.0\%] (Figure 4). The prevalence of intestinal parasitic infection in individuals with education level between high school to bachelor of science level, was 16.0\% [95\% CI:7.0\%-28.0\%] and in people with education higher than bachelorof science level was reduced to $12.0 \%$ [95\%CI:2.0\%-28.0\%] with no statistical significant difference between them $(\mathrm{Z}=0.41, \mathrm{P}=0.82)$ (Figure4). According to the results of random effect model, infection among male $(13.0 \%)$ was more than female $(8 \%)$ with statistically significant difference between them $(\mathrm{p}=0.00)$ (Figure 5). The results of meta-regression showed that the prevalence of intestinal parasitic infectionin food handlers have significantly decreased in recent years $(\mathrm{p}=0.01)$. Also, our analysis revealed that sample size had no effect on the prevalence of intestinal parasitic infection in food handlers ( $\mathrm{p}=0.68)$. To evaluate the effect of each study on the pooled prevalence, by meta-analysis, the sensitivity of studies was shown in Figure 2. At the first level a fixed effect meta-analysis was performed on 25 included studies and results revealed a considerable heterogeneity $\left(I^{\wedge} 2=99.40 \%, \mathrm{p}<0.001\right)$. In subgroup analysis, a random effect model was performed on parasite species (Figure 3). All effect sizes of 25 studies were located with95\% interval confidence. Therefore, studies had no effect on pooled prevalence of intestinal infections in food handlers and we can include all studies in meta-analysis (Figure2).

Food-borne parasitic diseases are one of main public health concerns all around the world whichmay lead to morbidity and mortality in developing countries (Simsek et al.2009). The importance of hygienic food preparation and delivery, revealthe importance of personal sanitation and education. This systematic review and meta-analysis aimed to evaluate the prevalence of intestinal parasitic infections in food handlers in Iran during 19 years (from 2000 to 2019). The results of meta-analysis revealed the overall prevalence of intestinal parasitic infections was 14\% [95\% CI: 11-17\%] in food handlers in Iran. The results indicated thepoor healthand inadequate personal hygiene in food handlers who are involve in food producing and food serving process in Iran. The highest rate (72\%) of infection was reported in a study carried out in East Azarbaijan by Garedaghi et al in 2014, and Dargahi et al. in 2016 who reported the rate of $59.4 \%$ in Tehran province (Garedaghi et al., 2014; Dargahi et al., 2016). The lowest prevalence of infection (1\%) was reported from Mazandaran province by Khazan et al in 2014 (Table 1). The sub-group analysis revealed that G. lamblia , with the prevalence of $41.0 \%$ [95\%CI: $25.0 \%-59.0 \%$ ], B. hominis, with $28 \%$ [95\%CI: $15.0 \%-44.0 \%$ ] and E.coli with $22.0 \%$ [95\%CI: $16.0 \%-29.0 \%$ ], had the highest prevalence among all intestinal parasites in food handlers in Iran. The highest rate of infection $(27.7 \%)$ was achieved in owners of school snack bars, where children took cooked food and snacks. The results may have a bias for small sample size, but the important point in this regard is that 5 of 18 different school cafeteria owners were infected with intestinal parasites whichis considerable. This may be resulted from weak health controlling programs in schools. In a study carried out by Costa- Cruz et al in Brazil, the researchers studied 20 school for the evaluation of intestinal parasitic infections in school food handlers. They found that, 49 of $104(47.1 \%)$ of school food handlers were 
infected (Khazan et al., 2013). Comparing their findings with ours indicates the higher rate of infection in their studied subjects. The meta-analysis revealed the high prevalence of intestinal parasitic infection in butchers $(11.5 \%)$ and backers $(10.17 \%)$. Theses two groups play an important role in public food health. Interestingly, the lowest prevalence of intestinal parasitic infection rate was observed among confectioners (1.70\%). Although the sample size comprised 978 cases and relatively big, the results indicate anappropriate personal hygiene in this group whichis regularly monitored by health care system.

In our meta -analysis, the infection rate of $13 \%$ in men achieved while the rate of infection among women was $8 \%$ with statistically significant difference ( $\mathrm{pi}$ 0.0001). Considering the higher rate of infection in men, the difference may be resulted from smaller sample size in women which is resulted from the fewer women involved in food handling than men in Iran. In some countries the ratio of men to women wasdifferent from ours. In a study in Thailand in 2011, Kusolsuk et al, studied 219 women and 47 men. This is resulted from the great role of women in food preparing and handling in Thailand. The result of their study revealed that infection rate in 273 food handlers was $10.3 \%$ which is higher than our results when compared with infection rate of $14 \%$ in 140447 subjects in our study. In contrast with our results, the most infecting cases were found with hookworms (70\%) while our most prevalent helminthic infection was with $H$. nana worms (Kusolsuk et al., 2013). Their results revealed the insufficient hygiene in food preparation and our results indicates inappropriate personalhygiene. Our meta-analysis showed that the highest intestinal infection in food handlers were caused by protozoan parasites and the most frequent parasite (41\%) was G. lamblia (Fig 3 ). These protozoa are considered one of the most pathogenic parasites (Arora et al., 2007) which can cause acute or chronic diarrhea with or without clinical signs. The parasite can be transmitted directly from infected persons to the healthy individuals, on the other hand eradication and controlling the parasite is very difficult.It is estimated that 200 million people in Asia, Latin America and Africa suffering from giardiasis (AL-Khayat et al., 2017).In a study carried out by Simsek et al in 2009 in Turkey, intestinal parasitic infection evaluated in 299 foodhandlers from Sanliurfa,Southeastern Anatolia. The results showed that $52.2 \%$ of food handlers were infected with intestinal parasites and most of them $(26.8 \%)$ were infected with G. lamblia, fallowed with A. lumbricoides (10.7\%) and T. saginata (10\%). Also $13.3 \%$ of them were infected with both Staphylococcus aureus and intestinal parasites. Unlike to our results, the infection rate withG. lamblia in their study was higher.

The meta-analysis elucidated that prevalence of intestinal parasitic infection in individuals with education level lower than high school, to be $20 \%$ while in individuals with education level between high school to bachelor of science level, was $16.0 \%$ and in cases with education higher than bachelor of science level was reduced to $12.0 \%$ but there found no statistical significant difference $(\mathrm{Z}=0.41, \mathrm{P}=0.82)$ (Figure 4). Although the results indicated no association between intestinal parasitic infection and educational levels, but it seems that infection rate in individuals with lower level is higher than those with higher educational levels. It seems that food hygiene knowledge, attitudes and practices in food handlers play an important role in prevention of food contamination with intestinal parasites. In a study designed by Acikel et al in 2008, a total of 83 food handlers in kitchen were evaluated with questionnaires for their information and behaviors before and after training. The results indicated the significant difference in behavioral practices and, the researchers concluded that education has an important impact on decreasing the infection rate in food handlers. Although the researchers studied the decreased bacterial density, it can be extended in parasitic infections too as the way of transmission is almost same (Acikel et al.,2008). In a study by Kheirandish et al, in 2011, out of 816 bakery workers with health certificate, 630 individuals had knowledge about intestinal parasitic infections and the ways of transmission but, 78(12.3\%) of them were infected with intestinal parasites. Also, 186(22.8\%) of this population had no knowledge in this regard and 19 (10.2\%) individuals were infected among them. These researchers declared that $85 \%$ of intestinal parasitic infections observed in people who did not attend in hygiene training programs. This shows that training in order to upgrade personal information in parasite transmission is necessary for all food handlers. Also training hygiene can affect the improvement of society health (Kheirandish et al., 2011).

\section{CONCLUSIONS:}


Our results revealed high prevalence of intestinal parasitic infection in food handlers in Iran. This high prevalence is largely due to poor personal hygiene practice, poverty, lack of knowledge, insufficient environmental sanitation and inadequate health controlling services.Although the food industryworkers, food handlers and anyone who is connected with the production, handling, storage, transportation, preparation, or else, is obliged to undergo routine medical examinations including stool microscopy for intestinal parasitic infections (once every 6 months) and chest $\mathrm{x}$ ray for tuberculosis suspected cases (occasionally). However, it seems that they are not sufficient. It is advised that some strict rules such as obligation in filling the stool container in the lab are added. Also, if infected food handler cases are identified, immediate decisions for exclusion of the career up the resolving all symptoms or completion of further investigations should be made. Additional programs including education for changing attitude about infectious diseases requires consideration.

CONFLICT OF INTEREST: The authors declare that they do not have any conflict of interest.

ETHICS APPROVAL : No ethical approval was required as this is a review article with no original research data.

\section{DATA AVAILABILITY STATEMENT:}

\section{REFERENCES}

Acikel CH, Ogur R, Yaren H, Gocgeldi E, Ucar M, Kir T. The hygiene training of food handlers at a teaching hospital.Food Control 2008; 19:186-190

AL-Khayat FA Al-Muhsin, Jameel SK, Wali MH. Public Health Importance of Some Common Intestinal Protozoa in Food Handler in Baghdad.I.J.S.N. 2017;8 (1): 26-34

Amiri A, Nazemi S, Raei M, Chaman R, Norouzi P (2013). A Comparison of Direct Technique and Formalin-Ether Method in Determining Parasitic Infection among Health-Card Applicants in Shahroud City. goums.ac.ir 7(3) [paper in Persian].

Arora, DR, Arora B. Medical Parasitology. 2nd ed. India, New Delhi: CBS publishers and Distributors 2007: 243

Asadi B, Ahi A (2011). Survey of these diseases - intestinal Artisans and food preparation and distribution centers and public places in the city Neishabour during year 1389. Congress, paper, Yazd, Iran [paper in Persian]

Babaei Pouya N, Akhlaghi L, Razmjou E (2018). Epidemiology of Intestinal Parasites among Applicants Receiving Health Card of Ardabil City in 2014. Journal of Health 9(1): 115-123

Balarak D, Jafari Modrek M, Ansari H. Prevalence of Intestinal Parasites among the Food Handlers in the City of Qom, 2014. Salamat Jmea, 2014;8 (4): 20-28

Balarak D, Modrek MJ, Bazrafshan E, Ansari H, KordMostafapour F (2016). Journal of Parasitology Research doi.org/10.1155/2016/8461965

Dargahi D, Zarebavani M,Einollahi N,Dashti N,Rezaeian M, Abbasi S. Prevalence of Giardia lamblia among Food Handlers and Day-Care Workers in Tehran. Payavard-Salamat.2016; 10(7): 402-408 [Article in Persian]

Davami MH, Kazaii MR, Rfii M, Milanii M (2006). An Investigation on The Prevalence of Intestinal Parasitic Infections in Food Handlers in Arak (Iran) During 2002-2003. Journal of Jahrom University of Medical Sciences 3(3) [Article in Persian]

Ebrahim, G., Publication bias in Meta-Analysis: prevention, assessment and adjustments Hannah R. Rothstein, Alexander J. Sutton, Michael Borenstein (eds) Chichester, John Wiley \& Sons Ltd. 2005. ISBN 0-470-870-141£ 55. 2006, Oxford University Press 
Esparar D, Belizario VY, Relos J. Prevalence of intestinal parasitic infections among food-handlers of a tertiary hospital in Manila using direct fecal smear and formalin ether concentration technique. The Philippine Journal of Microbiology and Infectious Diseases. 2004;33(3):99-103

Fallahizadeh S, Feiz-Haddad MH, Kazemi F, Afrisham R (2017). Prevalence of Intestinal Parasitic Infections in Shush County, Southwest of Iran during 2014 - 2016. International Journal of Infection 4(3):e14588

Fallah M, Sadeghyan S, Therkhani H, Habibi F, Heydar Barghy Z (2004). An Investigation on The Prevalence of Intestinal Parasitic Infections in food preparation and distribution centers and public places in the city Hamedan. Journal of Research in Health Sciences 1(7):3-10 [Article in Persian]

GaredaghiY, Firouzivand Y. Protozoan Infections of Restaurant Workers in Tabriz, Iran. Crescent Journal of Medical and Biological Sciences 2014; 1(2):46-48. Available in http://www.cjmb.org

Haraty Nejad Torbati AR, Khanjani N (2011). Epidemiological study of intestinal parasites in managers of food preparation, distribution and sales centers and public places from the beginning of 2008 to the end of 2010 in Rashtkhar city. https://www.civilica.com/Paper-NATURE01-NATURE01 131.html, Article code: COI, NATURE01_131. Congress in Iran, [Article in Persian]

Hatami H, Azarbarzin V (2018). The Prevalence of Intestinal Parasitic Infections in Food Handlers Health Applicants in Areas Covered by Shomal Health Center in Tehran in 2016. Community Health 5(1): ISSN 2423-4702

Heydari Hengami M, Hamedi Y, Najafi- Asl M, Sharifi-Sarasiabi Khojasteh (2018). Iranian Journal of Public Health 47(1), pp.111-118

Kassani A, Shaterian M, Sharifirad G, Menati R, Abbastabar H, Ebrahimipour M, et al. The prevalence of some intestinal parasites in food-handlers of Asian and African countries: A meta-analysis. Arch Hyg Sci $2015 ; 4(1): 49-56$

khazan H Halakou A.Prevalence of intestinal parasitic infections among food vendors

referred to Gonbad-e-kavus health central laboratories in 2013.Journal of Torbat Heydariyeh University of Medical Siences. 2014; 2(3): 57-61[Article in Persian].

Kheirandish F, Tarahi MJ, Haghighi A, Nazemalhosseini- Mojarad E, Kheirandish M. Prevalence of Intestinal Parasites in Bakery Workers inKhorramabad, Lorestan Iran. Iranian Journal of Parasitology 2011; 6(4):76-83

Kheirandish F, Tarahi MJ, Ezatpour B. Prevalence of intestinal parasites among food handlers in Western Iran. Revista do Instituto de Medicina Tropical de Sao Paulo. 2014;56(2):111-4

Koohsar F, Amini A, Ayatollahi A, Noshak G, HedayatMofidi H, Namjoo M (2012). The prevalence of intestinal parasitic infections in food handlers in Gorgan, Iran. Medical Laboratory Journal 6(1):26-34 [Article in Persian]

Kusolsuk T, Maipanich W, Nuamtanong S, Pubampen S, Sa-nguankiat S, Rojekittikhun W, et al. Parasitic and enteric bacterial infections among food handlers in tourist-area restaurants and educational-institution cafeterias, Sai-Yok district, Kanchanaburi province, Thailand. J Trop Med Parasitol 2011;34(2):49-53

Motazedian MH, Najjari M, Ebrahimipour M, Asgari Q, Mojtabavi S, Mansouri M. Prevalence of Intestinal Parasites among Food-handlers in Shiraz, Iran. Iranian journal of parasitology. 2015;10(4):652-7

Mohammadzadeh A, Spotin A, Mikaeili Galeh T, Fadaee M (2018). The prevalence of intestinal parasites in staff working at the restaurants of Tabriz city. Medical Journal of Tabriz University of Medical Sciences and Health Services 40(4): 60-66

Neghab M , Moosavi S, Moemenbellah-Fard M.D (2006). Prevalence of Intestinal Parasitic Infections among Catering Staff of Students' Canteens at Shiraz, Southern Iran. Pakistan Journal

of Biological Sciences 9 (14): 2699-2703 
Rokni MB. The present status of human helminthic diseases in Iran. Annals of tropical medicine and parasitology. 2008;102(4):283-95

Salary S, Safizadeh H (2013). Prevalence of Intestinal Parasite Infestation in the Food Suppliers of Kerman City, Iran, in 2010. Journal of Health \& Development 1(4) [Article in Persian]

Safi M, Tavalla M, Mardani M, Afrisham R (2016). Prevalence of intestinal parasitic infections among applicants for health cards attending Ahvaz East Health Center during 2012-2013. Asian Pacific Journal of Tropical Disease 6(2): 151-154

Sharif M, Daryani A, Kia E, Rezaei F, Nasiri M, Nasrolahei M (2015). Prevalence of intestinal parasites among food handlers of Sari, Northern Iran. Revista do Instituto de Medicina Tropical de São Paulo 57(2):139-144

Saki J, Khademvatan S, Masoumi K, Chafghani M (2012). Prevalence of intestinal parasitic infections among food handlers in Khuzestan, Southwest of Iran: a 10-year retrospective study. African Journal of Microbiology Research 6(10):2475-80

Simsek Z, Koruk I, Copur AC, Gürses G. Prevalence of Staphylococcus aureus and intestinal parasites among food handlers in Sanliurfa, Southeastern Anatolia. J Public Health Manag Pract. 2009 ;15(6):518-23. doi: 10.1097/PHH.0b013e3181aa2814

\section{Hosted file}

figures.docx available at https://authorea.com/users/343203/articles/469857-prevalence-ofintestinal-parasitic-infection-in-food-handlers-of-iran-a-systematic-review-and-metaanalysis

\section{Hosted file}

tables for transboundary.docx available at https://authorea.com/users/343203/articles/469857prevalence-of-intestinal-parasitic-infection-in-food-handlers-of-iran-a-systematicreview-and-meta-analysis 